\title{
Negative magnetophoresis in diluted ferrofluid flow
}

Cite this: DOI: $10.1039 / \times 0 \times x 00000 x$

Received 00th January 2012,

Accepted 00th January 2012

DOI: $10.1039 / \times 0 \times x 00000 x$

www.rsc.orgl

\author{
Majid Hejazian ${ }^{a}$ and Nam-Trung Nguyen ${ }^{a^{*}}$
}

We report magnetic manipulation of non-magnetic particles suspended in diluted ferrofluid. Diamagnetic particles were introduced into a circular chamber to study the extent of their deflection under the effect of a non-uniform magnetic field of a permanent magnet. Since the ferrofluid is a paramagnetic medium, it also experiences a bulk magnetic force that in turn induces a secondary flow opposing the main hydrodynamic flow. Sheath flow rate, particle size, and magnetic field strength were varied to examine this complex behaviour. The combined effect of negative magnetophoresis and magnetically induced secondary flow leads to various operation regimes, which can potentially find applications in separation, trapping and mixing of diamagnetic particles such as cells in a microfluidic system.

\section{Introduction}

The utilization of magnetic force for microfluidic applications has recently attracted a great attention from the research community. Magnetic force has been used in a variety of microfluidic applications such as pumping, separation, mixing, magnetowetting, manipulation of cells labelled with magnetic beads, and diamagnetic particles. ${ }^{1}$ Particle manipulation using magnetophoresis could be categorized into positive magnetophoresis and negative magnetophoresis. The later is also often called diamagnetophoresis. In positive magnetophoreis, magnetic particles migrate toward a higher magnetic field gradient. Magnetic particles or cells labelled with magnetic beads can be manipulated with this method. Diamagnetic particles could also be manipulated under relatively high magnetic field gradients due to the mismatching magnetic susceptibilities. The particles experience a repulsive force, and move away from the magnetic source. Using a paramagnetic medium, the repulsive effect can be achieved at a lower magnetic field strength and for micron sized objects. ${ }^{2,3}$ Considering the fact that most of the living cells possess diamagnetic properties, diamagnetophoresis could potentially be significant for label-free cell separation.

Concentrating diamagnetic particles using two repulsive or attracting magnets were experimentally and theoretically investigated by Wilbanks et $a l^{4}$ In this study, the effect of different magnet arrangements and flow rate on particle concentration were studied. Liang et $a l^{5}$ compared the migration behaviour of $2.85-\mu \mathrm{m}$ magnetic and $10-\mu \mathrm{m}$ diamagnetic particles in diluted (10\%) EMG 408 (Ferrotec) ferrofluid and in an aqueous solution. Using ferrofluid as the medium increases susceptibility mismatch and significantly improves the separation throughput. Peyman et al. ${ }^{6}$ used diamagnetic repulsion forces to demonstrate the potential applications in label-free manipulation of particles. Focusing, trapping and size selective diamagnetophoretic separation of diamagnetic particles were demonstrated. Zhu et al. ${ }^{7}$ employed an analytical model to study the trajectories of non-magnetic microparticles in ferrofluid through a microchannel in the proximity of a permanent magnet. This study concluded that a larger deflection could be achieved with larger particles or at a lower flow rate.
In many biological studies, cell concentration is an important step for sample preparation. Red blood cells are paramagnetic due to deoxygenated haemoglobin proteins, while other cells existing in blood are diamagnetic. ${ }^{8}$ Han and Frazier ${ }^{9}$ reported label-free separation of red and white blood cells from diluted whole blood with diamagnetic capture (DMC) mode and a high-gradient magnetic field. Their results showed that $89.7 \%$ of red blood cells and $72.7 \%$ of diamagnetic white blood cells could be separated. Rodriguez-Villarreal et al. ${ }^{10}$ investigated the effect of several parameters such as particle size, type and concentration of paramagnetic salt solution, as well as flow rate on focusing of diamagnetic polystyrene particles and living label-free HaCaT cells. This study revealed that raising the susceptibility mismatch between particles and carrying fluid, reduction of flow rate, increasing the particle size, and increasing residence time in the magnetic field can improve the focusing effect.

The use of a background ferrofluid enables the manipulation of non-magnetic particles and cells, as well as their biocompability has been investigated in the literature. Zeng et $a l .{ }^{11}$ also demonstrated magnetic concentration of particles and cells in a ferrofluid flow through a straight microchannel using permanent magnets. The concentration of both $5 \mu \mathrm{m}$ polystyrene particles and live yeast cells in 0.059 EMG 408 ferrofluid was investigated. The work examined the effect of flow rate, arrangement of the two attracting magnets and the magnet-to-magnet distance on the concentration performance and flow patterns. The authors discovered that dilute ferrofluid and magnetic force has negligible influences on the viability of yeast cells. Kose et al. ${ }^{12}$ reported separation of live red blood cells from sickle cells and bacteria. $40 \mathrm{mM}$ citrate concentration (stabilized with citric acid to yield a $\mathrm{pH}$ of 7.4) is found to be optimum for cell viability and ferrofluid stability combined. Au et al. ${ }^{13}$ investigated effects of nanoparticles on the adhesion and cell viability on astrocytes. The study suggests that the addition of nanoparticles to immature astrocytes causes inhibition of cell attachment and impedes subsequent growth. However, in mature astrocytes, nanoparticles induce mitochondrial uncoupling but do not alter cell membrane integrity. 


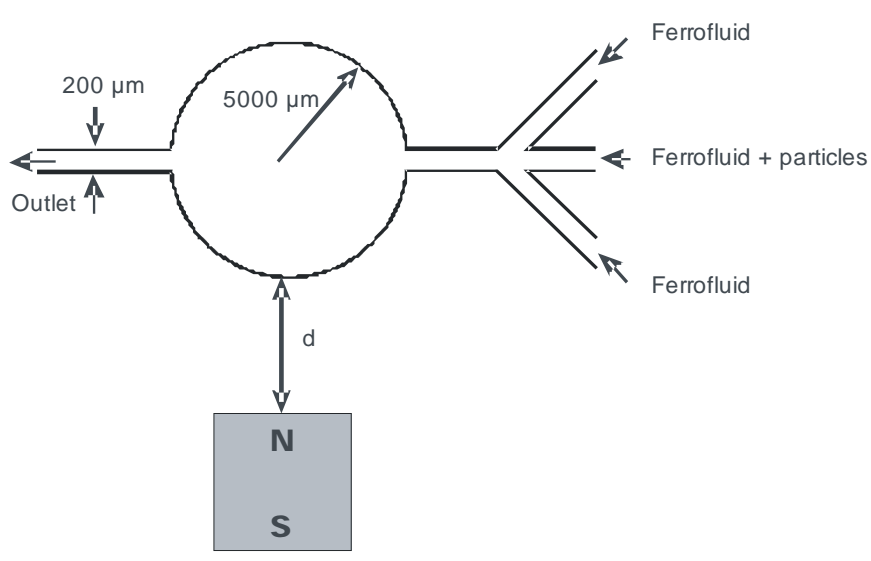

Fig. 1 Schematic of the microchannel and magnet setup.

In addition to separation, mixing is another important task in microfluidics. Mixing is required in many biochemical analyses such as cell activation, enzyme reaction and protein folding. Micromixers are categorized as passive and active types. In passive micromixers external energy is not needed, while active micromixers rely on an external field such as a magnetic field to induce mixing disturbances. ${ }^{14}$ Wang et al. ${ }^{15}$ studied the performance of a magnetic particle driven micromixer, consisting of a microchannel and a pair of electromagnets. Varying the design parameters such as applied magnetic actuation forces, they could demonstrate that the optimum switching frequency depends on the lateral dimension of the channel and the applied magnetic force. Roy et al. ${ }^{16}$ reported a droplet-based microfluidic system with magnetic stirring. The work investigated the impact on the overall mixing performance of parameters such as the rotational speed of the magnetic field, viscosity of the droplet liquid, and the concentration of magnetic particles in the liquid. Ganguly et al. ${ }^{17}$ examined in situ immunochemical binding of biotinylated oligonucleotides on streptavidin-coated magnetic beads through magnetophoresis-aided cross-stream mixing in a microfluidic channel. The effect of various ratios of the fluid streams and viscosities of the background fluid was evaluated on the extent of immunochemical binding. For the augmentation of mixing of a magnetic based reaction, Berenguel-Alonso et al. ${ }^{18}$ designed a simple and low-cost magnetic actuator, consisting of a CDshaped plastic unit with embedded magnets to trap and move the magnetic beads through a microfluidic chamber. Sensitivity was enhanced with the use of the magnetic actuator. The device was used for the amplification reaction of an enzyme-linked fluorescence immunoassay to detect Escherichia coli.

In our previous work, ${ }^{19}$ the migration of diamagnetic microparticles in a ferrofluid core stream sandwiched between two diamagnetic streams under a relatively weak and uniform magnetic field was investigated. Under the uniform magnetic field, provided by a custom made electromagnet, the core stream containing magnetic nanoparticles expanded into the surrounding diamagnetic streams. Diamagnetic particles suspended in the core stream also followed the same direction due to diamagnetophoresis effect.

Most of the works reported previously by others used relatively narrow and straight channels together with high field gradient generated by a permanent magnet. In this paper, we utilise the extreme combination of a large fluid chamber and a non-uniform and relatively stronger magnetic field generated by a permanent magnet. This unique combination creates the opportunity to investigate the effect of a high magnetic field gradient in relatively large chamber on deflection of nonmagnetic micro-particles. The numerical simulation predicted a deflection of the diamagnetic particles away from the magnet. However, experimental results showed a more complex phenomenon. We observed a secondary flow field in the upper half of the chamber opposite to the direction of the main hydrodynamic flow, as a result of the non-uniform magnetization of the ferrofluid. We first investigated the impact of field strength through changing the distance of the permanent magnet to the chamber, the sheath flow rate, and the size of the diamagnetic particles on the extent of the secondary flow. We then repeated the experiment with florescent dye, to prove that the secondary flow is caused by non-uniform magnetization of the ferrofluid. This interesting phenomenon has a potential to be used for both mixing and trapping/sorting of diamagnetic particles based on size.

\section{Materials and methods}

Fig. 1 shows a schematic of the microchannel and magnet setup. The circular chamber has two inlets and one outlet. The inlets and outlet channels have a depth of $H=50 \mu \mathrm{m}$ and a width of $W=200 \mu \mathrm{m}$. The circular chamber has a depth of $H=50 \mu \mathrm{m}$ and a diameter of $D=1 \mathrm{~mm}$. The microchannel was fabricated in polydimethylsiloxane (PDMS) using a standard soft lithography technique. Detailed information about fabrication procedure was reported in Song et $a .^{20}$ The moulded PDMS part was bonded to another flat PDMS base after treating both with oxygen plasma.

Two precision syringe pumps (SPM100, SIMTech Microfluidics Foundry) was used to deliver the fluids into the microfluic device. A camera (Edmund Optics, Germany) attached to an inverted microscope (Nikon Eclipse TE100) and connected to a desktop computer was used for visualization and image recording. The paramagnetic liquid used in our experiment is a water-based ferrofluid (EMG707, Ferrotec) diluted to $5 \%$ vol. of the off-the-shelf solution by DI water. Two solution samples were made by suspending 3.1 and 4.8 $\mu \mathrm{m}$ fluorescent polystyrene particles (Thermoscientific Inc.) in the diluted ferrofluid. The diluted ferrofluid without particles was introduced into the circular chamber (Fig. 1) as the sheath streams to focus the core stream with suspended particles. Fluorescein sodium salt (Acid Yellow, Sigma-Aldrich Co.) was used for tracing the core flow in a further experiment to confirm that the secondary flow is caused by the non-uniform magnetization of the ferrofluid, and the diamagnetophoresis does not play a significant role in the phenomenon.

A $3.2-\mathrm{mm}^{3}$ Neodymium-Iron-Boron (NdFeB) permanent magnet (B222, K\&J Magnetics Inc.) provided the magnetic field for this experiment. The PDMS device was cut to place the magnet on one side of the circular chamber. The experiments were carried out with three different distances (2, 3 and $4 \mathrm{~mm}$ ) between the magnet front and the edge of the chamber. The magnetic field of the permanent magnet was measured and calibrated using a Gaussmeter (Hirst Magnetic Instruments Ltd). Figure 2 shows the measured magnetic flux density as a function of the distance from the edge of the magnet. The stream containing particles was maintained at a constant flow rate of $1 \mu \mathrm{L} / \mathrm{min}$ throughout the experiments, 


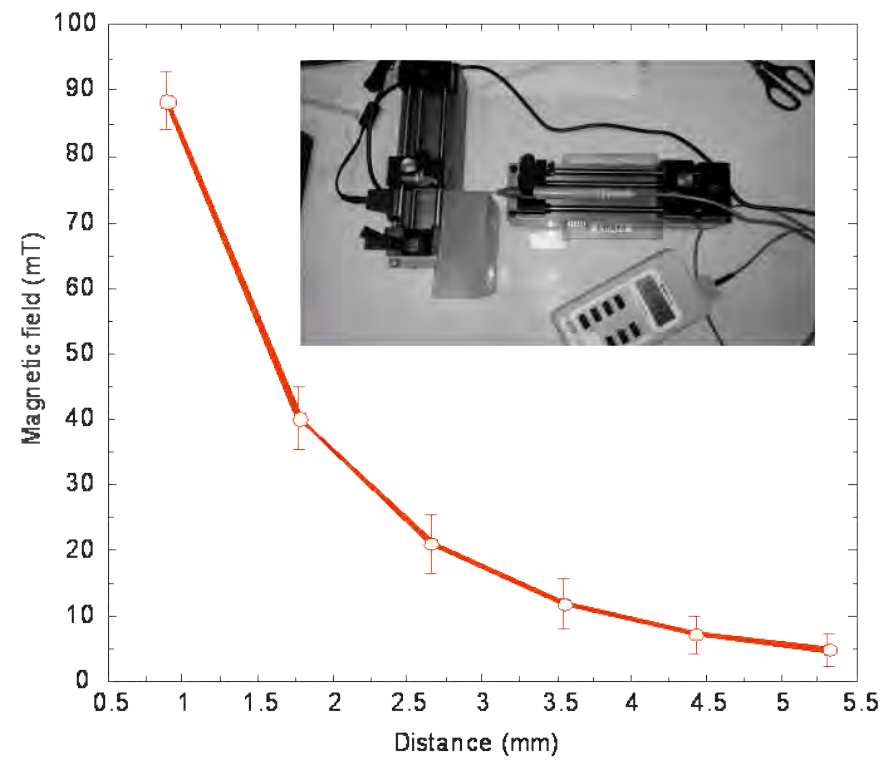

Fig. 2 Magnetic flux density versus distance to the permanent magnet. The inserted image shows the characterisation setup. The distance was adjusted using the linear stage of a syringe pump.

while the sheath flow rate was varied from 1 to $50 \mu \mathrm{L} / \mathrm{min}$ to observe the particle deflection and the secondary flow as a function of flow rate ratio and total flow rate.

\section{Theoretical background and numerical simulation}

A force balance should be considered to determine the trajectory of a particle in a magnetic field. In our study, diamagnetophoretic deflection of polystyrene mciroparticles is dominated by the drag force and the magnetic force. ${ }^{8}$ The magnetic force acting on a pointlike particle in a magnetic induction $\mathrm{B}$ can be estimated as: ${ }^{21}$

$\mathbf{F}_{m}=\frac{V \Delta \chi}{\mu_{0}}(\mathbf{B} \cdot \nabla) \mathbf{B}$

where $\mathbf{F}_{\mathrm{m}}$ is the magnetic force $(\mathrm{N}), V$ is the volume of the particle, $\Delta \chi=\chi_{p}-\chi_{f}$ is the magnetic susceptibility mismatch between particles $\chi_{p}$ and base fluid $\chi_{p}$ (dimensionless), $\mathbf{B}$ is magnetic flux density, and $\mu_{0}=4 \pi \times 10^{-7}\left(\mathrm{~T} \mathrm{~mA}^{-1}\right)$ is the permeability of the vacuum. The drag force for the suspended particles in a flow with relatively low Reynolds number can be determined using the Stokes' law and the relative velocity:

$\mathbf{F}_{f}=3 \pi \eta d_{p}\left(u_{f}-u_{p}\right)$

where $\eta$ is the dynamic viscosity of the fluid, $d_{p}$ is the diameter of the particles, and $u_{f}$ and $u_{p}$ are the velocities of the fluid and the particles, respectively.

Considering these dominating and most effective forces on particles, a numerical model for the prediction of particle trajectories could be established with the magnetic field, the single phase laminar fluid flow through the microchannel, and particle tracing in COMSOL (COMSOL Inc., USA). Our numerical model considers three distinct domains: the permanent magnet, the microfluidic channel and the surrounding PDMS substrate. A circular magnetic insulation boundary condition with the diameter of $2 \mathrm{~cm}$ was applied around the system, to bind the magnetic field and to achieve an accurate field distribution. The two-dimensional model consists of three inlets, a circular chamber and one outlet. The permanent magnet is considered as a square $(3.2 \mathrm{~mm} \times 3.2 \mathrm{~mm})$ placed in the distance of $2 \mathrm{~mm}$ from the edge of the chamber. The working fluid for all the inputs is ferrofluid diluted with DI-water (with volume fraction of 5\%), and considered as incompressible. Two streams were introduced into the chamber; the middle stream is set with a flow rate of $1 \mu \mathrm{L} / \mathrm{min}$, while the other two inlets for the sheath streams have equal flow rates varying between 1 to $7 \mu \mathrm{L} / \mathrm{min}$. Steady state condition is applied for simulation of magnetic field and fluid flow. The calculated flow and magnetic field was used to evaluate the trajectories of the particles using a time-dependent particle tracing method.

For modelling the laminar flow of diluted ferrofluid inside the microchannel, continuity equation:

$\nabla \cdot\left(\rho_{f} \cdot \mathbf{u}_{f}\right)=0$

and Navier-Stokes equations:

$\rho_{f}\left(\mathbf{u}_{f} . \nabla\right) \mathbf{u}_{f}=-\nabla P+\nabla \cdot\left(\eta \cdot \nabla \mathbf{u}_{f}\right)$

were solved, where $P$ is pressure, $\mathbf{u}_{\mathrm{f}}$ is fluid velocity, and $\rho_{\mathrm{f}}$ and $\eta$ are the density and dynamic viscosity of the fluid. The normal inflow velocities are set for the three inlets, no slip condition was considered at walls, and pressure without viscous stress at outlet.

A scalar magnetic potential were used to solve the magnetostatic problems, in the absence of electric currents:

$\nabla \cdot\left(\mu_{0} \mu_{r} \mathbf{H}\right)=0$

where $\mu_{\mathrm{r}}$ is the relative permeability, $\mathbf{H}$ is magnetic field strength $(\mathrm{A} / \mathrm{m})$. A magnetic insulation is considered around the whole system.

The magnetic susceptibility of various PS particles is $(7.93 \pm 0.16) \times 10^{-6} .^{22,23}$ Effective susceptibility of diluted ferrofluid emulsions could be estimated by Maxwell-Garnett formula: ${ }^{24}$

$$
\mu_{e f}=\mu_{w}+3 \mu_{w} \frac{\phi \frac{\mu_{p}-\mu_{w}}{\mu_{p}+2 \mu_{w}}}{1-\phi \frac{\mu_{p}-\mu_{w}}{\mu_{p}+2 \mu_{w}}}
$$

Where $\mu_{w}$ is the susceptibility of water, $\mu_{p}$ is the susceptibility of Polystyrene particles, and $\mu_{e f}$ is the effective susceptibility of diluted ferrofluid.

Particle trajectory were obtained by considering the balance of the dominant forces:

$m_{p} \frac{d \mathbf{u}_{p}}{d t}=\mathbf{F}_{m}+\mathbf{F}_{d}$

where $m_{\mathrm{p}}$ is the mass of the particle, $\mathbf{u}_{\mathrm{p}}$ is the velocity of the particle, $\mathbf{F}_{\mathrm{m}}$ is magnetic force on the particle, and $\mathbf{F}_{\mathrm{d}}$ is drag force. The initial position in which the particles are released is set to be the middle inlet, Fig. 1. Stick boundary condition $\left(\mathbf{u}_{\mathrm{p}}=0\right)$ was used for the microchannel walls and the outlet, meaning that the particles will stop when they reach the wall. The velocity field and the magnetic field in the flow domain, which has been calculated in the steady state study, were used to solve the force balance equation and to 

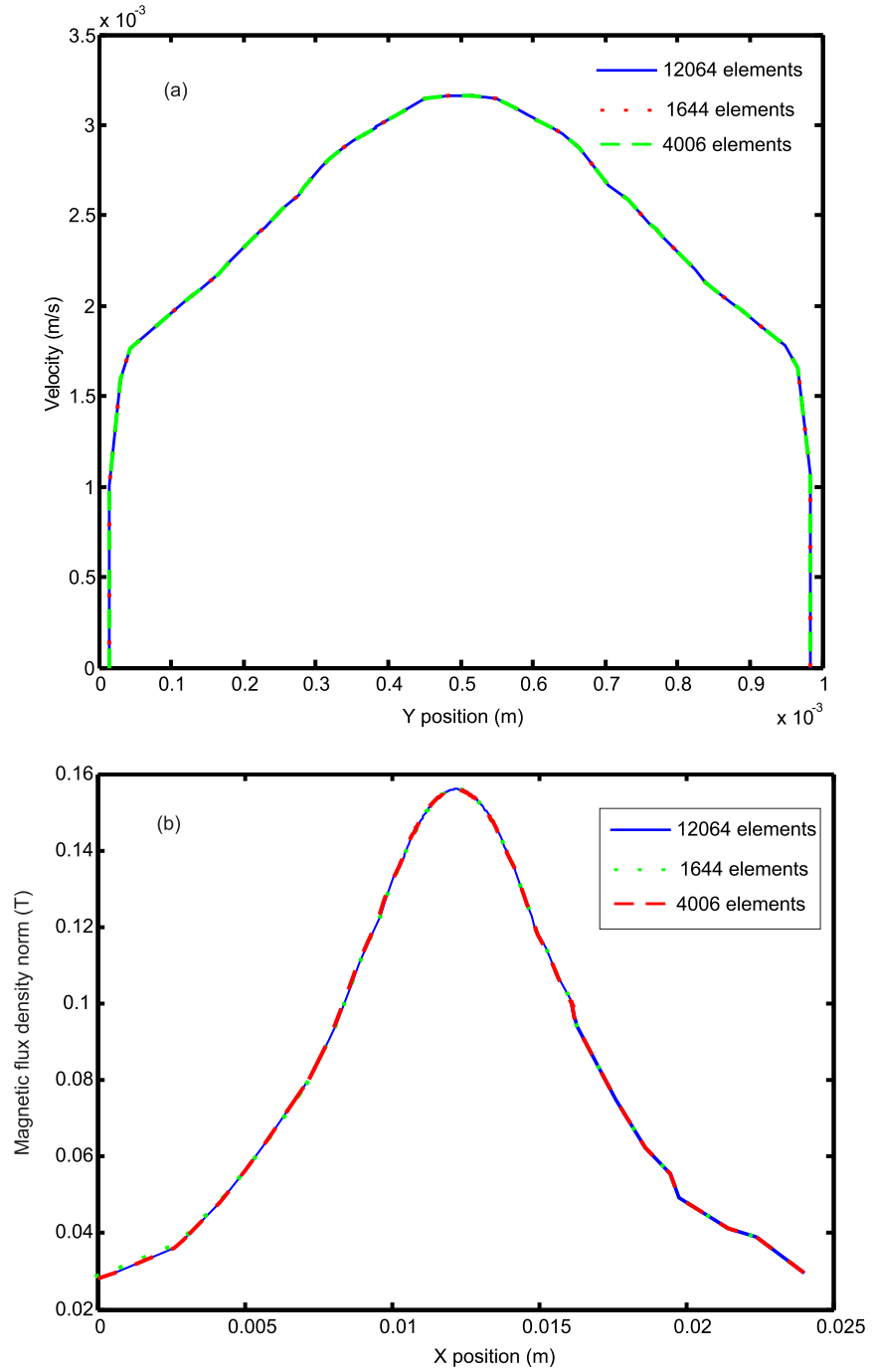

Fig. 3 Mesh independency test for (a) velocity profile; and (b) magnetic flux density norm.

predict the trajectory of the particles. The time step for the time dependent particle tracing study is set as $10^{-4} \mathrm{~s}$ to achieve an accurate and more detailed particle trajectory.

For the numerical solution of the above equations, finite element discretization was based on quadratic basis functions for magnetic field, second order functions for velocity, while the pressure field is described by linear basis functions. Figure 3 shows the grid independency for the simulation. Three structured non-uniform meshes (finer close to the walls where large gradients exist) have been evaluated for the velocity profile of pure water along a line parallel to the $y$-axis and crossing the centre of the chamber. Figure 3(a) shows that the velocity profile is not dependent on the number of elements. A test is also done for the magnetic flux density along a line parallel to the $x$-axis and crossing the centre of the chamber. Figure 3(b) indicates that the number of finite elements has no effect on the value of the magnetic flux density norm. The measured data from Fig. 2 deviates within 5\% from the simulated data shown in Fig. 3. In this study, 1644 elements were selected for meshing the geometry. Two-dimensional simulation was employed for this study, because of the flat geometry of the chamber requires much less computational time comparing to a three-dimensional (3D)

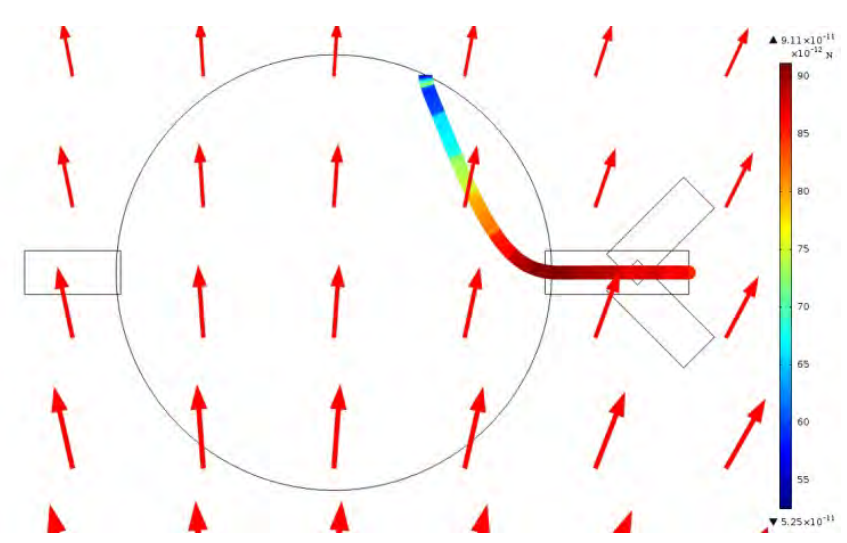

Fig. 4 The simulated magnetic field and the force distributions along a particle trajectory. The magnitude of the force is colour coded (4.8 $\mu \mathrm{m}$ particles, flow rate ratio of 1 ). The arrows show the magnitude of magnetic field.

simulation. The two-dimensional (2D) model considers the depthaveraged velocity.

The numerical simulation was carried out for two particle diameters (3.1 and $4.8 \mu \mathrm{m})$ and for various sheath flow rates between 1 and $15 \mu \mathrm{L} / \mathrm{min}$, while the flow rate of the core flow containing the particles is held constant at $1 \mu \mathrm{L} / \mathrm{min}$. The results are illustrated in Fig. 5. As can be seen from these trajectories, the deflection for the diamagnetic particle increases with decreasing the sheath flow rate and increasing particle size.

\section{Results and Discussions}

In our experiments, the non-uniform magnetic flux was exerted on the system using the permanent magnet at variable distances of 2, 3 and $4 \mathrm{~mm}$ to the edge of the chamber. In order to study the effect of sheath flow rate on the deflection, the particle flow is held constant while the sheath flow varies between 1 and 50 $\mu \mathrm{L} / \mathrm{min}$. Two particle sizes, 3.1 and $4.8 \mu \mathrm{m}$, were used to study the impact of particle size on deflection of the particle stream. The experimental results are summarized in Fig. 6 and Fig. 7.

\section{Effect of sheath flow rate}

The first interesting observation at low flow rates is the existence of a secondary flow opposing the main hydrodynamic flow. The secondary flow can be seen in the upper half of the chamber, and circulation of particles in some locations is also apparent. We hypothesize that this phenomenon is induced by the non-uniform magnetization of the ferrofluid, because of the non-uniform magnetic field distribution across the chamber. Due to magnetic attraction, the magnetic nanoparticles migrate towards the magnet. The magnetic nanoparticles of the ferrofluid accumulate in the lower half of the chamber, while the nanoparticels in the upper half is blocked by the core flow. Because of this blockage, a higher concentration of magnetic nanoparticle particle exists just above the core flow. As an example, for flow rate ratio of 7 and magnet distance of $2 \mathrm{~mm}$, the core flow is expanded into the upper half of the chamber, Fig. 6(a). As the flow rate ratio decreases, this difference in the concentration of the ferroparticles causes a flow in the opposite direction of the main flow, strong enough to compete with the upper sheath flow, and appears as a secondary flow. On the other hand, the particles near the edge of the florescent particle 


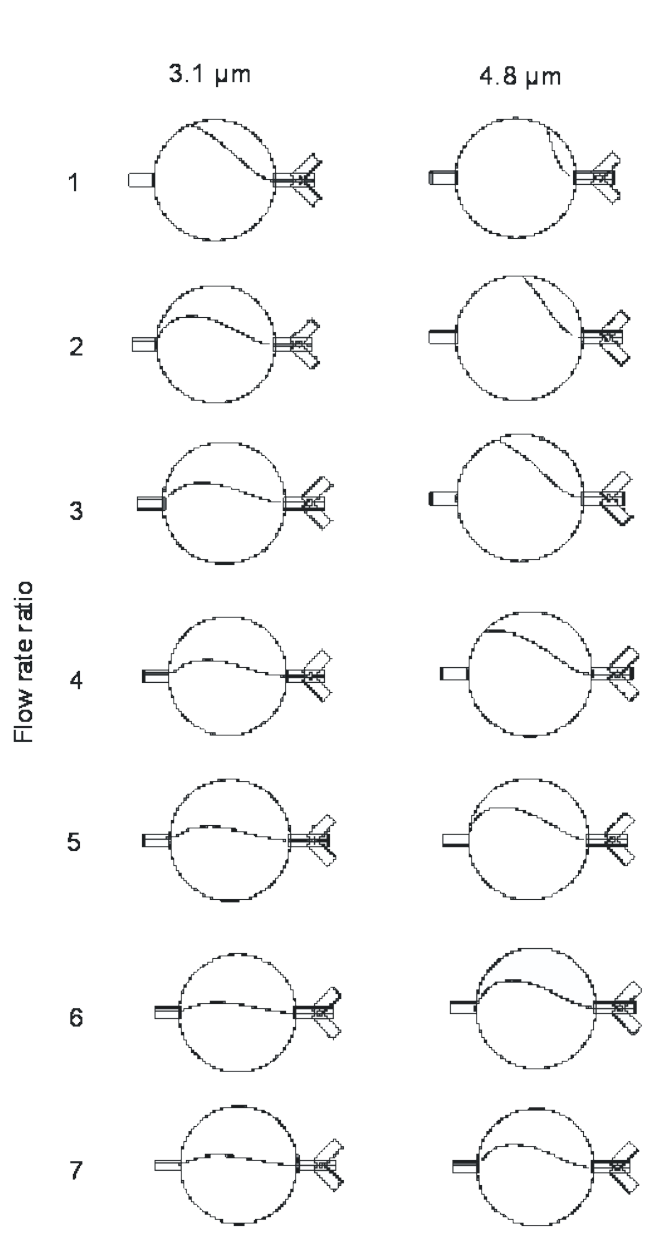

Fig. 5 Simulation results showing the variation of magnetic deflection in different sheath flow rates for one particle only (distance $2 \mathrm{~mm}$ ).

stream, at low sheath flow rates, depart from the core stream, because of the diamagnetophoresis effect, and are dragged into the secondary flow path. As the sheath flow rate increases, this secondary flow starts to fade away. The numerical simulation was unable to model and demonstrate the secondary flow, because a uniform concentration was considered for the base flow throughout the system.

Another noticeable phenomenon is that the deflected particles do not follow a focused line. Instead, a wide band of particles is observed. The deflected core flow is not integrated and it could be divided into two streams; the lower one is the main stream and flowing to the chamber outlet, while the upper and less dense band of particles travels a longer path to reach the outlet. The particles in the upper band are more deflected and travel in a lower velocity compared to the particles in the lower band. This behavior occurs because of the velocity distribution in the $z$-axis along the height of the chamber, which has its maximum in the center and decreases when approaching the walls. The extent of diamagnetic deflection depends on the balance between magnetic force in $y$-direction and hydrodynamic force in $x$-direction. The particles closer to the middle of the chamber height have a higher velocity and less deflection, because of larger hydrodynamic force. This behavior also fades with increasing sheath flow rate.

The S-shape of the particle trajectory also deviates from the ideal case of the numerical simulation. Near the entrance, the

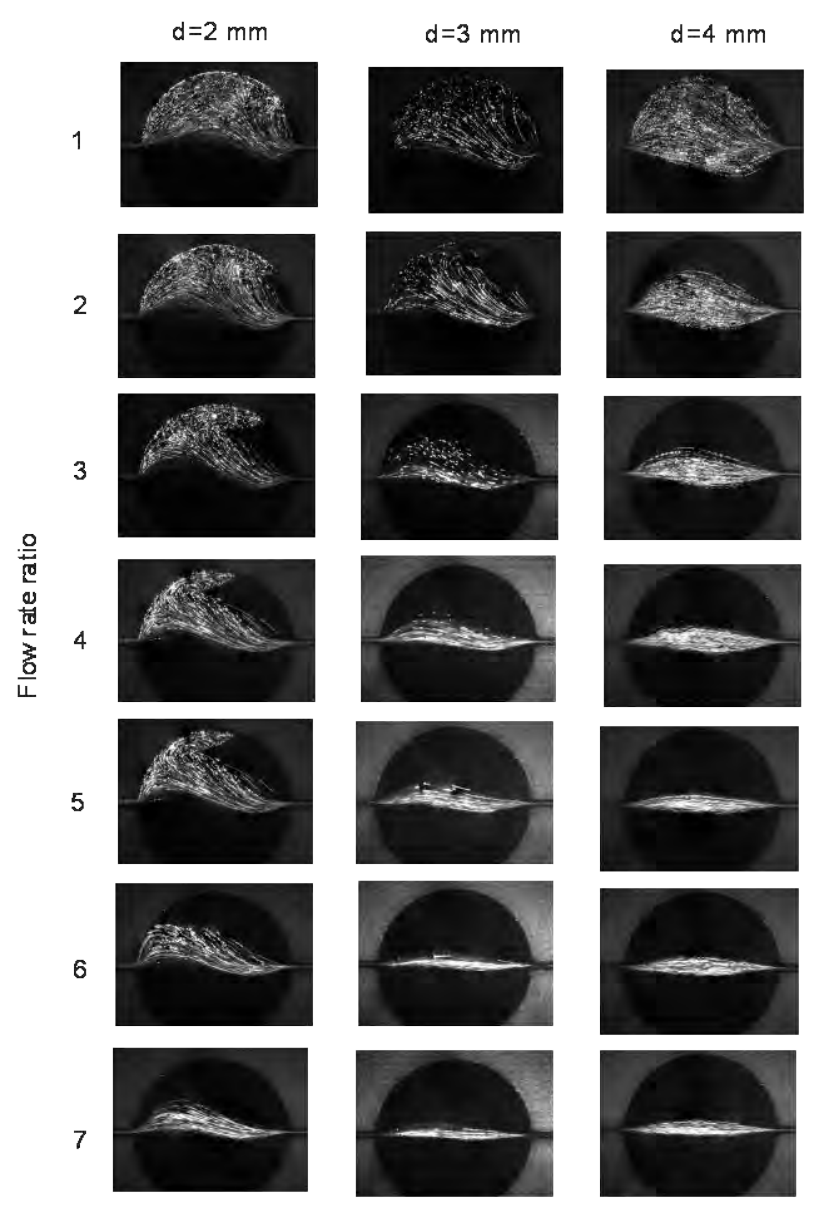

Fig. 6 Experimental results of 3.1- $\mu \mathrm{m}$ particles for three different magnet-to-chamber distances $(2,3$, and $4 \mathrm{~mm})$.

trajectory bends downwards, while close to the outlet it bends upwards as expected with the simulation (Fig. 5). The downward bend is caused by the sudden change in concentration of magnetic nanoparticles due to the downward migration discussed above.

\section{Effect of magnetic field strength}

The experiment was repeated for three different positions of the permanent magnet, 2, 3, and $4 \mathrm{~mm}$ from the edge of the chamber to investigate the effect of magnetic field strength. The results are shown in Fig. 6 and Fig. 7. Considering the fact that the secondary flow opposes the main hydrodynamic flow, the upper sheath flow competes with the secondary flow induced by the magnetic field. A weaker magnetic field leads to a weaker magnetization of the ferrofluid. Therefore the secondary flow appears at lower flow ratios and a shorter magnet distance. Also, by decreasing the magnetic field strength, the difference between the upper and lower bands of the deflected particles becomes less apparent, and the stream of particles becomes more uniform. The reason lies in the diamagnetophoresis effect. A lower magnetic field leads to weaker magnetic force and less particle deflection, therefore the core stream mainly follows the flow path caused by the magnetization of the ferrofluid.

\section{Effect of particle size}

The experiments were carried out with two particle sizes (3.1 and $4.8 \mu \mathrm{m})$ to observe the effect of particle size. For the small particles, at flow rate ratio of 1 and magnet to chamber distance 


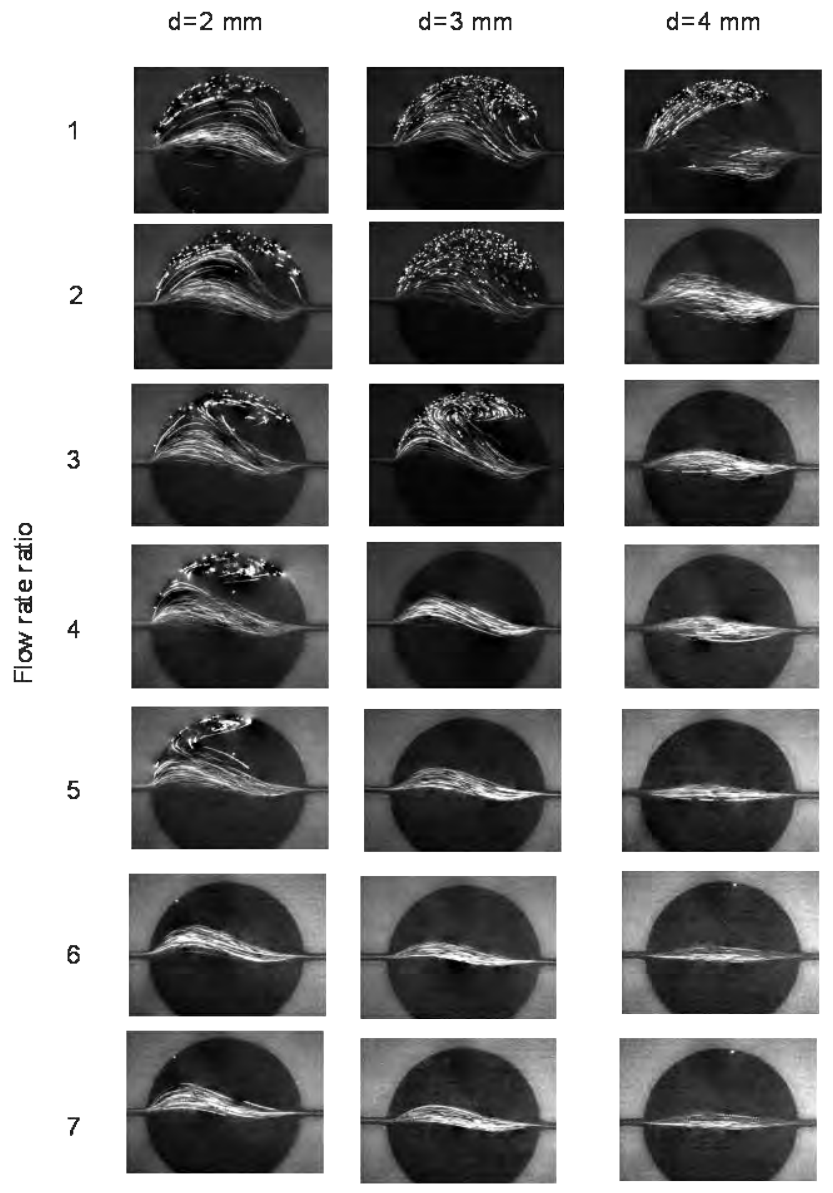

Fig. 7 Experimental results of $4.8-\mu \mathrm{m}$ particles for three different magnet-to-chamber distances $(2,3$, and $4 \mathrm{~mm})$.

of $4 \mathrm{~mm}$, the effect of secondary flow on particles could be avoided. Meanwhile, larger particles under the same conditions still show significant secondary flow. Having smaller particle size leads to a lower hydrodynamic force caused by secondary flow according to equations (2), and therefore the small particles exit the chamber unaffected by the secondary flow.

The flow ratio of 6 was taken for comparison to compare the effect of particle size on the particle band width. The images in Fig. 6 and Fig. 7 show that small particles cause more spreading and a wider band. The reason can be justified by the fact that larger particles are more concentrated in the middle of the channel, and less in the vincinity of the channel walls. Therefore, larger particles have a larger mean velocity that leads to a narrower particle band.

In our experiment, the diamagnetophoresis effect could not be distinguished clearly because of the existence of the secondary flow. However, it can be noticed by comparing the extend of deflection for the bottom of the particle stream, between two particle sizes. At low flow rate ratios (less than 6) more deflection could be observed at the bottom of the particle streams. Fig. 8 shows an operation pam the range of conditions, in which secondary occurs.

\section{Experiment with fluorescein dye}

We repeated the experiments using fluorescein sodium salt dissolved in ferrofluid in the core stream without

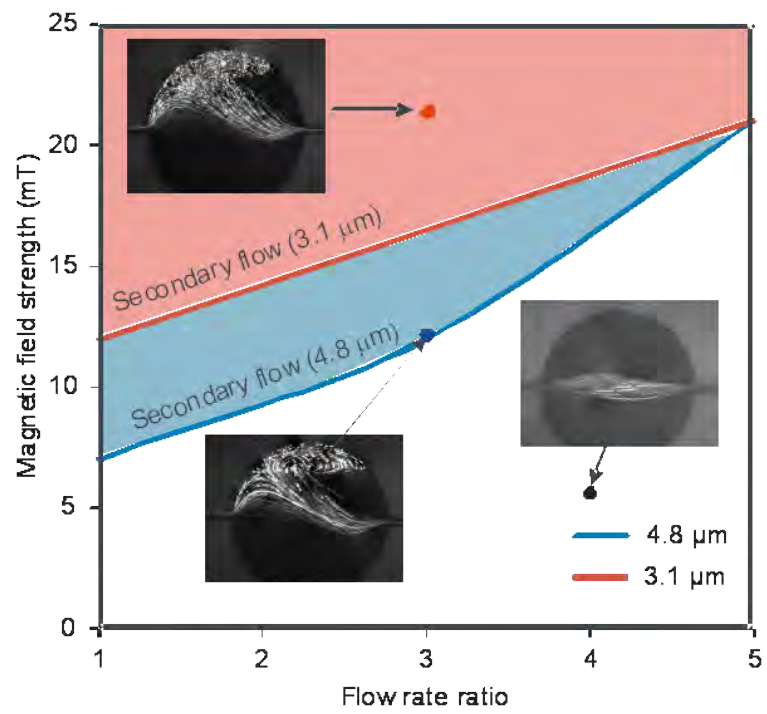

Fig. 8 Operation map for the secondary flow. The shaded areas are conditions with the secondary flow.

microparticles. The purpose of this experiment is to prove that negative magnetophoresis plays no role in the observed recirculation of the particles, as they just follow the induced secondary flow. Figure 9 shows the fluorescent image of the chamber under the same experimental conditions of Fig. 6 and Fig. 7. The results show that the secondary flow occurs at almost the same conditions as observed previously with the particles. A deflection of fluorescent dye towards the upper half of the chamber could be observed. The secondary flow and mixing of the fluorescent dye become stronger with decreasing sheath flow rates. The S-shape deflection could also be observed in Fig. 9. The downward migration near the inlet is caused by the ferrofluid concentration difference in that location, as the magnetic nanoparticles move towards the magnet. The upward migration happens before the chamber outlet as the result of the secondary flow.

\section{Conclusions}

This paper reports the behaviour of non-magnetic particles in diluted ferrofluid under the effect of a magnetic field in a circular chamber. The effect of sheath flow rate and magnetic field strength was examined for two particle sizes and fluorescein dye. Interestingly, a secondary flow in the opposite direction of the main flow was observed. At low flow rate ratios and relatively high magnetic field strengths, the secondary flow is strong enough to mix the core flow with the upper sheath flow. Results from the experiment with fluorescein dye prove that the secondary flow is caused by the non-uniform magnetization of ferrofluid, and the non-magnetic particles mostly follow the path of this flow. This phenomenon has potential applications for particle or cell capture based on their size difference and also for magnetic mixing of two streams in microfluidic chamber. A possible approach for simulating the secondary flow observed and reported in this paper is described as follows. The migration and consequently the concentration distribution of magnetic nanoparticles can be modelled by adding the magnetophoretic migration term to the convective/diffusive transport equation as reported previously in by our group. ${ }^{25}$ The distribution of magnetic susceptibility of the ferrofluid can then be determined based on the concentration distribution. The term of bulk magnetic force is 


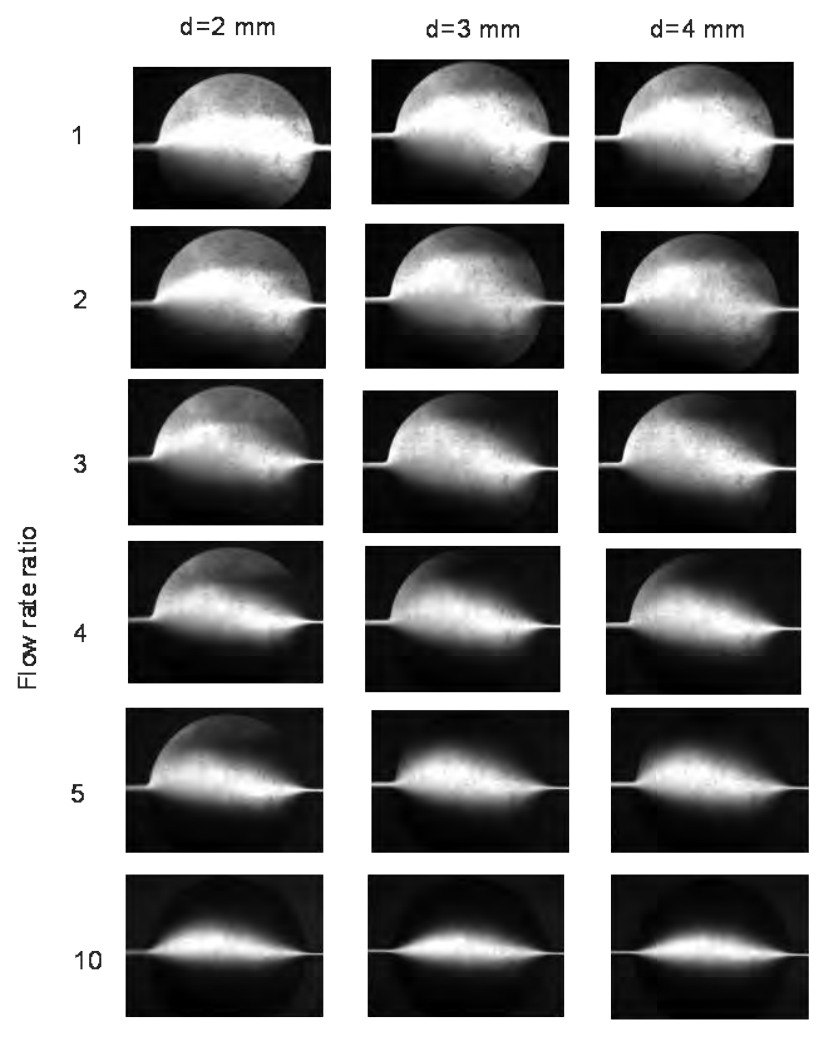

Fig. 9 Experimental results of fluorescent dye for three different magnet-to-chamber distances $(2,3$, and $4 \mathrm{~mm})$.

then added to the momentum equation (4), which can then be solved iteratively together with mass conservation equation and the convective/diffusive transport equation.

\section{Acknowledgements}

The authors acknowledge the start-up grant of Griffith University to NTN and the international PhD scholarship to MH.

\section{Notes and references}

${ }^{a}$ Queensland Micro and Nanotechnology Centre, Griffith University, Brisbane, 4111, Australia. E-mail: nam-trung.nguyen@griffith.edu.au; Fax: +6107373 58021; Tel: +610737353921

1. N.-T. Nguyen, Microfluid Nanofluid, 2012, 12, 1-16.

2. H. Watarai and M. Namba, Journal of Chromatography A, 2002, 961, 3-8.

3. Y. Chen, P. Li, P.-H. Huang, Y. Xie, J. D. Mai, L. Wang, N.-T. Nguyen and T. J. Huang, Lab on a Chip, 2014, 14, 626-645.

4. J. J. Wilbanks, G. Kiessling, J. Zeng, C. Zhang, T.-R. Tzeng and X. Xuan, Journal of Applied Physics, 2014, 115, 044901-044907.

5. L. Liang, C. Zhang and X. Xuan, Appl. Phys. Lett., 2013, 102, 234101-234104.

6. S. A. Peyman, E. Y. Kwan, O. Margarson, A. Iles and N. Pamme, Journal of Chromatography A, 2009, 1216, 9055-9062.

7. G. P. Zhu, N. T. Nguyen, R. Ramanujan and X. Y. Huang, Langmuir, 2011, 27, 14834-14841.

8. M. Hejazian, W. Lib and N.-T. Nguyen, Lab Chip, 2015, 15, 959.

9. K.-H. Han and A. B. Frazier, NSTI-Nanotech 2005, 2005.

10. A. Rodríguez-Villarreal, M. Tarn, L. Madden, J. Lutz, J. Greenman, J. Samitier and N. Pamme, Lab Chip, 2011, 11, 12401248 .
11. J. Zeng, C. Chen, P. Vedantam, T.-R. Tzeng and X. Xuan, Microfluid Nanofluid, 2013, 15, 49-55.

12. A. R. Kose, B. Fischer, L. Mao and H. Koser, PNAS, 2009, 106, 21478-21483.

13. C. Au, L. Mutkus, A. Dobson, J. Riffle, J. Lalli and M. Aschner, Biol Trace Elem Res, 2007, 120, 248-256.

14. N.-T. Nguyen and Z. Wu, Journal of Micromechanics and Microengineering, 2005, 15.

15. Y. Wang, J. Zhe, B. Chung T. F. and P. Dutta, Microfluid Nanofluid, 2008, 4, 375-389.

16. T. Roy, A. Sinha, S. Chakraborty, R. Ganguly and I. K. Puri, Physics of Fluids, 2009, 21, 027101.

17. R. Ganguly, T. Hahn and S. Hardt, Microfluid Nanofluid, 2010, 8, 739-753.

18. M. Berenguel-Alonso, X. Granados, J. Faraudo, J. AlonsoChamarro and M. Puyol, Anal Bioanal Chem, 2014, 406, 66076616.

19. G.-P. Zhu, M. Hejiazan, X. Huang and N.-T. Nguyen, Lab on a Chip, 2014, 14, 4609-4615.

$20 . \quad$ C. Song, N. T. Nguyen, S. H. Tan and A. K. Asundi, Lab Chip, 2009, 9, 1178-1184.

21. M. A. M. Gijs, F. Lacharme and U. Lehmann, Chem. Rev., 2009, $110,1518-1563$.

22. M. K. Moraveji and M. Hejazian, Numerical Heat Transfer, Part A, 2014, 66, 315-329.

23. H. Watarai, H. T. T. Duc, T. T. N. Lan, T. Zhang and S. Tsukahara, Anal Sci., 2014, 30, 745-749.

24. A. Sihvola and I. Lindell, Helsinki University of Technology, $1989,43,1989-1921$ 